\section{EL SENDERO DE LA REGULACIÓN BANCARIA Y LA CRISIS FINANCIERA INTERNACIONAL}

\author{
THE PATH OF THE BANK REGULATION AND INTERNATIONAL \\ FINANCIAL CRISIS \\ Julio Alberto Hennings Otoya $\left(^{*}\right)$ \\ Docente Auxiliar de la Facultad de Ciencias Contables - UNMSM \\ [Recepción: Agosto de 2011/ Conformidad: Octubre de 2011]
}

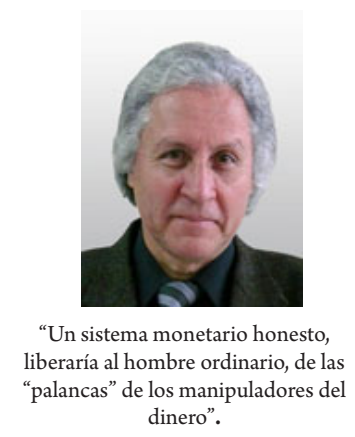

BENIAMIN FRANKLIN

\section{RESUMEN}

El escenario financiero mundial se encuentra en serias dificultades. La crisis originada en el país más ¿rico? de la tierra, cuya explosión del 2007 en el sector de las hipotecas norteamericanas hizo que antiguas y modernas instituciones bancarias, de seguros, de inversión y relacionadas, algunas consideradas "muy grandes para quebrar”, pasaran a la historia como ejemplo doloroso de la falta de un manejo prudente de los riesgos, los que cada vez son mas diversos y amplificados por el proceso globalizador que recorren los mercados. Aquí, es importante notar, que la administración de las instituciones bancarias de los EE.UU., en su afán de "crear valor", ergo ganar más para satisfacer a sus accionistas y sobre todo "cubrir las apetencias" desmedidas de muchos directivos, se vio incentivada paralelamente por las medidas laxas del gobierno de EE.UU, que socavaron las posibilidades de supervisión de sus entidades financieras y del control racional del sistema en su conjunto.

Este artículo describe y analiza los antecedentes de la regulación bancaria, el papel cumplido por el Banco de Pagos Internacional y los resultados del Comité de Supervisión Bancaria a través de los acuerdos de Basilea, dejando entrever la omisión de su aplicación por parte de las entidades norteamericanas.

Palabras claves: Crisis financiera, regulación bancaria, Banco Internacional de Pagos (BIS), Acuerdos de Basilea.

\begin{abstract}
The world financial situation is in serious difficulties. The crisis originated in the country more Are you rich? land, whose explosion in 2007 in the U.S. mortgage sector made ancient and modern banking institutions, insurance, investment and related, some considered "too big to fail", would pass into history as a painful example of the lack a prudent risk management, which are becoming more diverse and amplified by the globalization process that run the markets. Here, it is important to note that the administration of U.S. banking institutions, in an effort to "create value", ergo earn more to satisfy their shareholders and above all to "meet the desires" inordinate of many managers, was stimulated in parallel by the lax measures the U.S. government, which undermined the possibilities for monitoring their financial institutions and the rational control of the whole system.

This article describes and analyzes the history of banking regulation, the role played by the Bank for International Settlements and the results of the Committee on Banking Supervision by the Basel Accords, suggesting the omission of its implementation by U.S. institutions .
\end{abstract}

Keywords: Financial crisis, bank regulation, Bank for International Settlements (BIS), Basel Accords.

* Docente Investigador de la Facultad de Ciencias Contables - UNMSM, Docente Auxiliar de la Facultad de Ciencias Contables, UNMSM, Doctor en Ciencias Económicas - UNFV. E- mail: jhennings@speedy.com.pe 


\section{INTRODUCCIÓN}

En la mitad del siglo XIX, las operaciones bancarias en el mundo eran relativamente similares a las actuales, aunque los bancos tenían un carácter más local. Sin embargo, existía un tremendo riesgo en la Banca: Los Bancos comerciales realizaban negocios en el Mercado de Valores, estos en forma paralela a su función de captadores y colocadores de capital.

Por esa lejana época, en los EE.UU. con la expansión de los ferrocarriles, la industria del acero y el petróleo, los Bancos estadounidenses crecieron y se fortalecieron. Por su parte, la tradicional Banca Inglesa iba asentándose en Nueva York, con miras a aprovechar la ebullición de los nuevos negocios.

Probablemente este escenario, no percibía oportuno crear una regulación y un banco central, dada además, la popular aversión a un control centralizado que existía en la nación norteamericana. Respecto al gobierno, se decía que contaba con el apoyo de financistas privados como JP Morgan, para en caso necesario, estabilizar la economía nacional.

Recién en 1904 y con el gobierno de Theodore Roosevelt, empieza la preocupación estadounidense por la regulación de la economía, suscitándose por aquellos tiempos varios acontecimientos importantes, como:

- En 1908 se crea la Comisión Monetaria Nacional

- En 1911 se da paso a las leyes "Cielo Azul” que regulaban la venta de títulos dentro de un estado, realizadas por emisores fuera de ese estado

- En 1910 Nelson Aldrich presentó una propuesta para crear un Banco Central que fue ley para diciembre 23 de 1913.

- El Federal Reserve Actde 1913 establece el Sistema de la Reserva Federal, que reguló los bancos, y creó 12 Bancos Federales Regionales
Para ser realistas, ya desde antes de 1913, los Estados Unidos de América habían experimentado algunos "pánicos bancarios".

Al iniciarse la primera conflagración mundial, EEUU manteniéndose al margen de la guerra, asume el papel de abastecedor de bienes $y$ de capitales, lo que facilita que su Banca y Mercado de Valores adquirieran una gran dimensión. Cuando finaliza la Primera Guerra Mundial, el gran país del norte era ya el centro financiero del mundo.

Entre 1922 y 1929 en paralelo al alto crecimiento trimestral de su economía (con excepción de 1923); se desata una desmedida especulación de la banca en los mercados de valores, lo que empujaría al país a la gran depresión de 1929, momento quehace evidente la necesidad de implementar reformas sustanciales al sistema.

Así, de 1933 a 1940 en la administración del "New Deal" de Franklin D. Roosevelt, con ideas inspiradas en los postulados de J.M. Keynes, nace la "regulación bancaria de los Estados Unidos, a través de TheGlassSteagallAct, la cual divide las actividades de banca comercial y banca de inversión, no pudiendo los primeros tener la posesión de acciones de empresas, excepto en condiciones inusuales como la bancarrota, evitando con esto la posibilidad de especulación y mejorando su control.

Este hecho, trae de acompañamiento en los siguientes años, una sucesión de compras, fusiones y alianzas estratégicas en la banca americana, dejando atrás la estructura fragmentada y de carácter local, y también, consolida alas grandes organizaciones con operaciones globales. Se puede afirmar, que esta experiencia también contribuyó a que se dieran procesos similares en Japón y Reino Unido.

Este avance, desde un punto de vista racional y acertado para facilitar la regulación y 
posterior supervisión de la banca estadounidense, se comienza a cuestionar en los80s, durante el gobierno republicano de Ronald Reagan. En orden con las ideas de Milton Friedman, se argumentaba que la alta competencia mundial hacía que la Glass-Steagall quedase desfasada e "innecesaria". Consecuentemente, la Reserva Federal empieza a flexibilizar las regulaciones que separaban los dos negocios, hasta que finalmente en 1998 la Glass-Steagall Act es derogada por la Gramm-Leach Acten, paradójicamente con partida de defunción en un gobierno demócrata, el de Bill Clinton.

A partir de aquí, los bancos pasan a ser por "la competencia", nuevamente compañías de "Full Service", permitiendo la consolidación de actividades, e incrementando el riesgo de la banca tradicional (comercial), por efecto de su participación en actividades paralelas de especulación.

\section{EL PAPEL DEL BANCO INTERNACIONAL DE PAGOS}

A fin de explicar mejor las relaciones de la banca y las finanzas actuales, debemos mencionar que un elemento importante dentro de la arquitectura financiera mundial lo constituyó la creación del Banco Internacional de Pagos (Bank of International Settlements). La creación del Banco se dio en 1930 en la ciudad de Basilea (Suiza), en el contexto del Plan Young, que lidiaba con el tema de los pagos indemnizatorios impuestos sobre Alemania por el Tratado de Versalles. A partir de su fundación el Banco fue cambiando

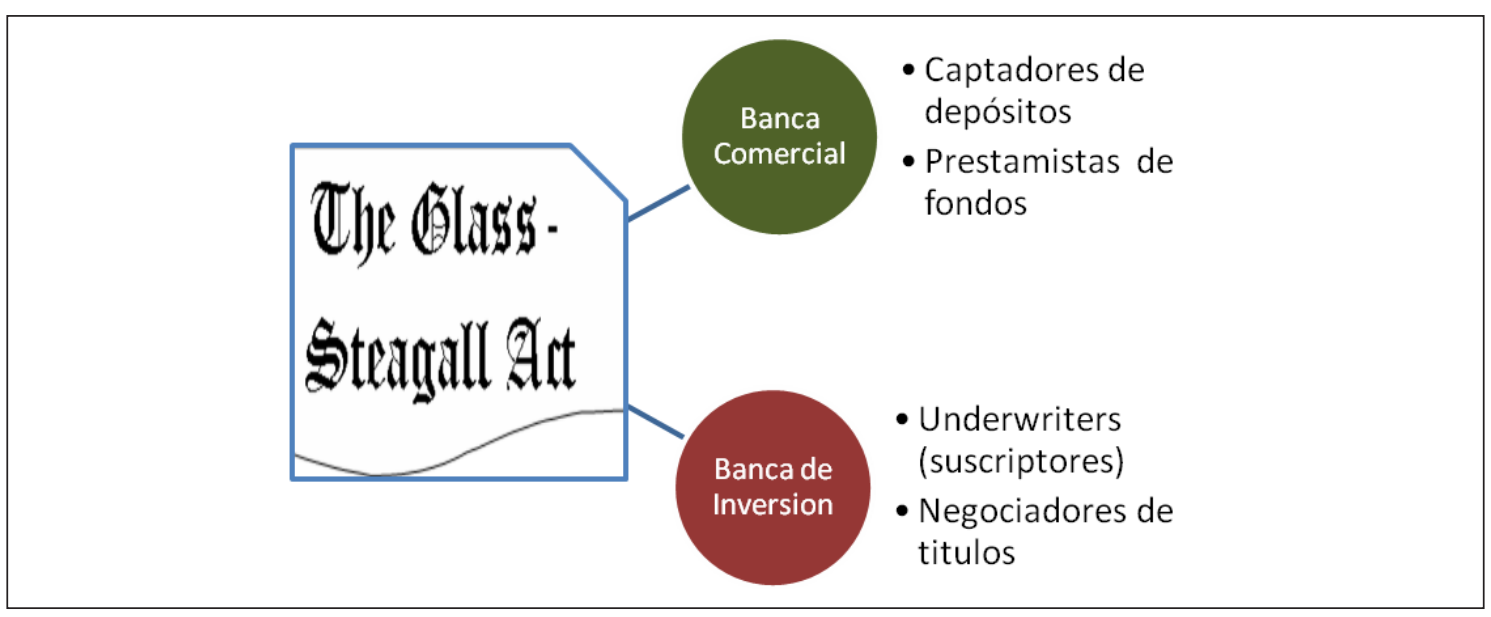

progresivamente su rol para convertirse en un importante mecanismo de coordinación y cooperación entre Bancos Centrales y Supervisores Bancarios.

Después de la II Guerra Mundial, la banca privada particularmente de los países desarrollados, buscó y generó su expansión. Así, la banca comercial en Francia se desarrolló notablemente al igual que los bancos italianos, alemanes, suizos, holandeses, ingleses, suecos y daneses. Paralelamente a esto, la banca norteamericana fue ejemplo de ayuda internacional y se consolidó entre los años sesenta y setenta como los pioneros de la banca corporativa, ejemplos notables fueron el Chase Manhattan Bank, First Nacional City Bank (hoy Citigroup),Manufacturers Hannover Trust, Bank of América... etc. etc.

En este ambiente, el Banco Internacional de Pagos (BIS) en los 70's implementó y defendió el sistema de Bretton Woods. En los 70s y 80s, se concentró en el manejo transfronterizo de los flujos de capital que siguieron a las crisis del petróleo y de la deuda 
internacional. Las crisis también pusieron sobre la mesa el tema de la regulación y supervisión bancaria.

Actualmente, el BIS cuenta con 58 bancos centrales miembros, todos los cuales tienen el derecho de ser representados y de votar en las reuniones generales. Sin embargo, el poder real y administrativo es desproporcionado a favor de algunos, por ejemplo su Consejo de Administración, posee seis miembros natos que son los líderes de los bancos centrales de Bélgica, Francia, Alemania, Italia, Reino Unido y Estados Unidos, los cuales pueden nombrar un representante adicional por cada uno de dichos países, quedando según estatuto, elegir un número máximo de nueve gobernadores de los demás países miembros, -por una mayoría de dos tercios-, para completar el numero de Consejeros (el Consejo actual tiene 18 miembros). A la fecha, los elegidos pertenecen a Canadá, China, Japón, México, Suecia, Suiza, y el Presidente del Banco Central Europeo.

\section{EL COMITÉ DE BASILEA Y LA DÉCADA PERDIDA DE AMÉRICA LATINA}

Teniendo como anfitrión al BIS, en diciembre de 1974, los gobernadores delos bancos centrales de las economías más desarrolladas (G-10) crearon el Comité de Supervisión Bancaria de Basilea, con el fin de mejorar la colaboración entre las autoridades de supervisión bancaria y generar estándares mínimos que sean de obligatorio cumplimiento para los países miembros.El G-10 en realidad está integrado por once países: Alemania, Bélgica, Canadá, EEUU, Francia, Italia, Japón, Países Bajos, Reino Unido, Suecia y Suiza, y concentró gran parte de la economía mundial.
Este Comité ha venido constituyendo un foro de debate para la resolución de problemas específicos de supervisión. Coordina la distribución de las competencias supervisoras entre las autoridades nacionales, a fin de garantizar una supervisión eficaz de las actividades bancarias, y con el paso del tiempo, si bien no posee ninguna autoridad de supervisión sobre los países miembros y sus conclusiones no tienen fuerza legal, ha formulado una serie principios y estándares de supervisión bancaria que han sido acogidos no solo por los países miembros, sino por la mayoría de países en el mundo.

Entrada la década de los ochenta, llamada la década perdida de América Latina, muchos bancos norteamericanos poseían excedentes en dólares, llamados comúnmente petrodólares, para ser colocados fuera de su país. Empezaron y se dispersaron los préstamos con intereses blandos para ciento de empresas y entidades financieras de casi toda América Latina. Simultáneamente a esto, la banca europea también prestaba para proyectos en la región, pero fueron más conservadores y desde mediados de los años ochenta se aglomeraron los más importantes bancos de Europa Occidental para crear desde la ciudad de Basilea, las primeras normas para fortalecer cualquier institución financiera.

\section{¿POR QUÉ REGULAR LA ACTIVIDAD BANCARIA?}

En este punto, es bueno aclarar que no existe consenso entre los tratadistas y expertos en banca, sobre los motivos que puedan justificar la necesidad de regular la actividad bancaria. Algunos consideran, que este sector debería regirse simplemente por las reglas y la disciplina del mercado, y que las intervenciones de los estados sobre ellas, no 
hacen sino perjudicar la competencia, que finalmente afecta al mismo mercado. Otros, como Fernández Baca (2004), esgrimen poderosas razones para regular la actividad bancaria, tales como:

1. La protección de los depositantes: al respecto, la mayor parte de legislaciones internas de los países buscan proteger los ahorros del público, y esto podría constituir el objetivo más importante en pro de la regulación de los bancos. En nuestro medio, el artículo 87 de la Carta Magna prescribe que: "El estado fomenta y garantiza el ahorro. La ley establece las obligaciones y los límites de las empresas que reciben ahorros del público, así como el modo y los alcances de dicha garantía". A continuación, establece que la Superintendencia de Banca y Seguros (SBS) ejerce el control de las empresas bancarias.

2. La necesidad de preservar la red de pagos de la economía: el mercado bancario pertenece a un tipo especial de mercado y los servicios bancarios, tienen unas características particulares (Shy 2001), como:

- Complementariedad, compatibilidad y estándares. Los bancos producen una serie de servicios que se complementan, como la utilización de tarjetas afiliadas a VisaElectron, o las de tarjetas de crédito mundiales; asimismo, sus servicios están compatibilizados bajo un mismo estándar, es decir están coordinados con el Banco, incluyendo los cargos, comisiones, cobro de intereses etc.

- Las externalidades de consumo. Los beneficios percibidos por cada consumidor depende directamente del número de usuarios del servicio, así por ejemplo el beneficio de un cliente de banco crece a medida q' aumenta el número de personas y de establecimientos afiliados al sistema bancario, con los cuales por ejemplo, pueda emitir cheques, realizar transferencias bancarias etc.

- Economías de escala. Es una característica común de las industrias que tienen costos fijos muy altos, como los bancos. En la medida que extiendan su red bancaria, su capacidad y costo para proporcionar liquidez $y$ servicios de pago a sus clientes mejorará.

Otro aspecto a tomar en cuenta en lo concerniente a la regulación bancaria, lo constituye el ámbito diverso de las actividades bancarias, las que son supervisadas por los entes gubernamentales, y que se pueden dividir en ocho grandes áreas:

a. La red de seguridad del gobierno

b. Las restricciones sobre las tenencias de activos y los requerimientos de capital de los bancos

c. El otorgamiento de licencias a los bancos y la supervisión prudencial

d. Los requerimientos de información

e. La protección a los consumidores

f. Las restricciones a la competencia

g. La separación del negocio bancario del negocio de valores

h. La coordinación internacional de la regulación bancaria

De los anteriores, los esfuerzos normativos originados por el Comité de Basilea, materia del presente estudio, a fin de promover la estabilidad financiera mundial se ha centrado en el tratamiento de los factores de riesgos de los bancos, enfocándose inicialmente en los requerimientos de capital según el nivel de riesgo de sus activos, para después, en sus versiones Basilea II y Basilea III incluir otros factores cruciales en el tema, como la supervisión prudencial y la trasparencia de información que generan los bancos, además de reforzar más las previsiones de capital. 
El sendero recorrido por los "acuerdos de capital" de Basilea hasta la fecha, se han visto confrontados con concurrentes crisis financieras y económicas mundiales, las cuales muy probablemente hubiesen afectado a más bancos y dañado con mas severidad el sistema bancario internacional, sino hubiesen estado vigentes.

\section{ACUERDO DE CAPITAL DE BASILEA (BASILEA I)}

Las pautas recogidas en el I Acuerdo de Basilea, o sea Basilea I de 1988, de adecuación de capital para la banca, han sido de trascendente importancia. Las reglas en la práctica, demostraron su valía, sobretodo la principal, por la cual los bancos deben mantener un volumen de capital mínimo equivalente al menos al $8 \%$ del valor total de sus activos, ponderado por su nivel de riesgo.

En 1994 explotó una crisis financiera que hizo que muchos de los bancos norteamericanos, pequeños y medianos, que prestaron dinero por toda América Latina colapsaran, siendo absorbidos por bancos más grandes y teniéndose que negociar las liquidaciones de los préstamos con emisiones de bonos denominados Brady, denominación alusiva al responsable de la Secretaría del Tesoro de los Estados Unidos de entonces, James Brady. Este, dio el respaldo para poder recuperar a mediano y largo plazo, las colocaciones efectuadas. De esta crisis, resultó el famoso y sonado efecto tequila de la quiebra de bancos en México. Otro ejemplo notable fue el caso de Venezuela en 1994, con la quiebra de 16 bancos y la emisión de más de US\$ 8 mil millones en bonos de la República.

A partir de estos eventos, los bancos fueron capitalizándose paulatinamente para soportar en 1997 la crisis financiera asiática, al igual que las seguidas crisis rusa, brasile- ra y de Turquía. El acuerdo de Basilea I sin duda, jugó un papel importante en el fortalecimiento de los sistemas bancarios. La repercusión de ese acuerdo, en cuanto al grado de homogenización alcanzado en la regulación de los requerimientos de solvencia fue extraordinaria.

Mientras Basilea I fue diseñada para bancos con actividad internacional y para los entonces 11 países representados en el Comité de Basilea, sin embargo, más de 150 países lo adoptaron. Además, contó con el reconocimiento del Fondo Monetario Internacional y del Banco Mundial como buena práctica internacional.

En este punto, conviene mencionar que el Acuerdo de Capital de Basilea (Basilea I) detalla las reglas para determinar los requerimientos mínimos de capital que las instituciones bancarias debían cumplir. En este sentido, Basilea I propuso una metodología para medir el riesgo crediticio según la estructura de activos mantenido por una entidad bancaria. Dicha medida consideraba el capital de una institución bancaria como proporción de la suma de los activos del banco ponderados por un factor que refleje el riesgo crediticio de dicho activo. Se acordó que el estándar mínimo de esta proporción sería de 8\% para proteger a los depositantes del riesgo de insolvencia del banco.

\section{Efectos de Basilea I}

Si bien Basilea I significó un gran avance en el proceso de supervisión bancaria, sin embargo, su planeamiento no fue suficientemente sensible al riesgo. Bajo su aplicación, no necesariamente se cumple el principio de a mayores riesgos mayores cargas de capital, a fin de cubrir dicho riesgo.

Lo anterior a su vez podría significar un incentivo para las instituciones financieras a tomar mayores riesgos, ya que estos no vendrían acompañados de mayores exigencias 
de capital regulatorio. Reconociendo esta deficiencia, el Comité de Basilea I empezó la tarea de reformar Basilea I y generar un nuevo esquema más sensible al riesgo.

\section{NUEVO ACUERDO DE CAPITAL (BASILEA II)}

De hecho, un sistema bancario con suficientes provisiones de capital es fundamental para "capear" las tormentas temporales del clima económico. Se deduce que las economías de Norteamérica y Europa no hubieran podido sobrevivir a muchas tensiones económicas-y hasta eventos como el 11 de Septiembre en New York-, sin haberse visto muy afectados, de no haber sido por la acumulación de capital que realizaron durante la etapa de expansión previa. Sin embargo, la desregulación de la banca norteamericana en las últimas dos décadas, permitiéndole ejercitar actividades de banca de inversión, ha ocasionado que los riesgos involucrados se multipliquen aceleradamente, propiciando el descontrol en las funciones esenciales de los bancos comerciales, conduciendo a muchas instituciones tradicionales al descalabro económico a partir de la crisis inmobiliaria de los EE.UU. y cuyos graves coletazos, aun no culminan en la escena internacional.

En los mercados emergentes, como los de América Latina y Asia, que a menudo han enfrentado situaciones de mayor volatilidad, las normas sólidas sobre adecuación de capital han sido y son probablemente muy importantes.

El paisaje financiero actual ha cambiado significativamente con respecto a las décadas anteriores. Se ha registrado un proceso de liberación, innovación y globalización. Así pues, los nuevos instrumentos financieros minimizaron las restricciones existentes y las hicieron más sencillas de soslayar. Así pues, los mercados financieros globales se ido integrando cada vez más.

El nuevo Acuerdo de Capital o Basilea II empezó a mediados de los años 90 y finalizó en junio de 2004, se aprobó un Nuevo Acuerdo de Capital (Basilea II) para los países miembros del G-10, el mismo que empezó a regir en dichos países a partir de 2007 en sus versiones mas simples y a partir de 2008 en sus versiones mas avanzadas. El objetivo principal de este Nuevo Acuerdo era lograr una mayor alineación de los requerimientos de capital de las entidades financieras con los verdaderos riesgos que éstas enfrentan y, a la vez, tomar en cuenta en el esquema de supervisión la evolución de las técnicas de manejo de riesgo y la creciente complejidad y heterogeneidad del sistema financiero.

Más allá de proponer metodologías más sensibles al riesgo para el cálculo del capital regulatorio, Basilea II planteo reglas prudenciales específicas para las instituciones de crédito. Diseñado y construido sobre la base del acuerdo de 1988, el Nuevo Acuerdo de Capital (NAC) apuntaba a incentivar la estabilidad del sistema financiero dando mayor importancia a los sistemas de control interno, a la administración de los bancos y a la disciplina de mercado.

Es así que Basilea II se podía definir como un marco global de supervisión bancaria, basado en tres pilares:

i. Los Requerimientos Mínimos de Capital (Con ponderaciones de riesgo asignadas a los diferentes tipos de activos de riesgo. Adicional al riesgo crediticio y de mercado que venia considerándose en Basilea I, se incluye aquí, los riesgos operacionales).

ii. El Proceso de Examen del Supervisor (Supervisión corriente por parte de las entidades reguladoras, asegurando que cada banco cuente con procesos inter- 
nos confiables para evaluar la suficiencia de su capital. Se establece también que aquellos riesgos no considerados en el primer pilar pueden incluirse como requerimientos adicionales de capital -a discreción del supervisor).

iii. La Disciplina de Mercado (basado en el supuesto de que los participantes de mercado bien informados recompensarán una estrategia consciente de administración riesgo en sus decisiones de crédito e inversión y penalizarán un comportamiento más arriesgado).

\section{Efectos de Basilea II}

Luego de un breve análisis sobre los efectos notorios por la adopción de Basilea II, sobre todo para los países de menor desarrollo, que dependen más del financiamiento externo, nos permite decir que el beneficio que trajo en general fue:

- Creación de incentivos para mejorar los procedimientos de evaluación de riesgos.

- Mejoras en el sistema de gobierno corporativo. Se formularon nuevos modelos internos de riesgo.

- Cambio cultural: Concientización de la dirección en la administración del riesgo.

- Surgimiento de nuevas y mejores herramientas de información financiera, y actualización y adecuación de normas contables (NIIF's).

- Las necesidades de información hizo que se produjeran nuevas inversiones en tecnología.

\section{BASILEA III: MARCO REGULADOR GLOBAL PARA REFORZAR LOS BANCOS Y SISTEMAS BANCARIOS}

Basilea III, surgió en medio de otra crisis financiera internacional, esta vez iniciada en el mundo desarrollado, los EE.UU del año 2007, y cuya conclusión está aun no está escrita. Así, al ponerse de relieve una vez más la urgente necesidad de fortalecer la regulación, supervisión y gestión de riesgos del sector bancario, el Comité de Basilea ${ }^{1}$ acordó el marco de Basilea III en septiembre del 2009, publicando las propuestas concretas vía documentos consultivos, en diciembre del 2009. Estos documentos constituyen la base de la respuesta del Comité a la crisis financiera y forman parte de las iniciativas mundiales para fortalecer el sistema de regulación financiera propuestos por los líderes del G-20 (Group of Central Bank Governors and Heads of Supervision).

Las medidas planteadas en Basilea III están encaminadas a:

i. Mejorar la habilidad del sector bancario para absorber los shocks resultantes de un estrés económico o financiero.

ii. Mejorar la gestión de riesgos y la gobernabilidad.

iii. Fortalecer la trasparencia de las entidades financieras y la publicación de información relevante.

Los objetivos de la reforma tienen dos enfoques:

i. La regulación a nivel de banco (regulación micro prudencial), lo cual contribuirá a incrementar la resistencia de las instituciones bancarias individuales a periodos de estrés.

ii. La regulación macro prudencial, protegiendo al sistema bancario del riesgo sistémico ante el incremento de los créditos durante periodos cíclicos expansivos.

Estos dos enfoques son complementarios a la supervisión en la medida que una mayor resistencia de los bancos individuales reduce el impacto de los shocks sobre todo el sistema.

Para resumir, se puede afirmar que los principales cambios de Basilea III respecto 
al acuerdo anterior, se da en el paquete de reformas orientado al incremento del capital requerido, la introducción de un estándar global de liquidez y el aumento de la sensibilidad al riesgo, dentro de un proceso de mejora de los sistemas de administración de riesgo de la banca mundial.

La combinación de una definición de capital más rigurosa, mayores requerimientos mínimos y la introducción de nuevos colchones de capital hará que los bancos estén en mejor situación de afrontar periodos de tensión económica y financiera, coadyuvando con ello el crecimiento económico.

\section{CONCLUSIONES}

1. Los "acuerdos de capital" de Basilea hasta la fecha, se han visto confrontados con concurrentes crisis financieras y económicas mundiales, las cuales muy probablemente hubiesen afectado a más bancos y dañado con mas severidad el sistema bancario internacional, sino hubiesen estado vigentes y o no hubiesen sido implementadas por muchas instituciones bancarias y supervisores de los diversos países, como fue el caso del Perú y otros países emergentes.

2. Asimismo, se considera que la aplicación de Basilea III terminará por fortalecer el sistema bancario internacional, pero sus efectos benéficos, es probable que no puedan ser disfrutados por muchos de los países pobres, donde no poseen una banca local fuerte y donde mayormente requieren de apoyo crediticio.

3. En este orden global de ideas, si bien teóricamente no constituye una obligación apegarse a los enfoques básicos de Basilea III, la práctica y el tiempo lo harán necesario como lo fue Basilea I y Basilea II. Aunque en esto, como dijo Heinrich ${ }^{2}$ refiriéndose a Basilea II: "La discrecionalidad nacional incorporada en Basilea II, ofrece a los supervisores tomar en cuenta las experiencias y realidades económicas en sus países".

4. Con relación al cumplimiento de las reglas de Basilea, resulta muy dudoso que los Bancos de los países desarrollados donde se generó y amplificó la crisis financiera internacional actual, las hayan tomado en consideración. La primera y cuyo principio responde a una lógica elemental para operar en el mercado "Los Requerimientos Mínimos de Capital", no fue satisfecha por el alto grado de apalancamiento que mantenían muchos intermediarios, entre ellos instituciones centenarias y prestigiosas, participando en la emisión de instrumentos, colocación y propia demanda de créditos "especulativos", -y añadiría irracionales. Detrás de este festín de inversiones, como pudo comprobarse después, había muchos títulos (iactivos?) sin valor y con solo un respaldo imaginario.

5. Igualmente, según las normas de Basilea II, las entidades reguladoras del sistema deberían asegurar que cada banco cuente con procesos internos confiables para evaluar la suficiencia de su capital, esto no se dio en los acontecimientos previos a la crisis hipotecaria. La FED del gobierno americano por evitar el desplome de la burbuja inmobiliaria disminuye el interés (ver grafico de John Taylor ${ }^{3}$ ) y

1 El Comité de Supervisión Bancaria de Basilea lo integran altos representantes de autoridades de supervisión bancaria y bancos centrales de Alemania, Arabia Saudita, Argentina, Australia, Bélgica, Brasil, Canadá, China, Corea, España, Estados Unidos, Francia, Hong Kong RAE, India, Indonesia, Italia, Japón, Luxemburgo, México, los Países Bajos, el Reino Unido, Rusia, Singapur, Sudáfrica, Suecia, Suiza y Turquía, y sus reuniones las celebran en la sede del Banco Internacional de Pagos (Suiza). 
acepta el relajamiento de requisitos para otorgar créditos a personas de dudosa solvencia (subprime). Esto nos hace inferir, que hubo en el mejor de los casos desidia cuando no complicidad de los organismos reguladores del sistema, no cumpliendo con el principio aludido.

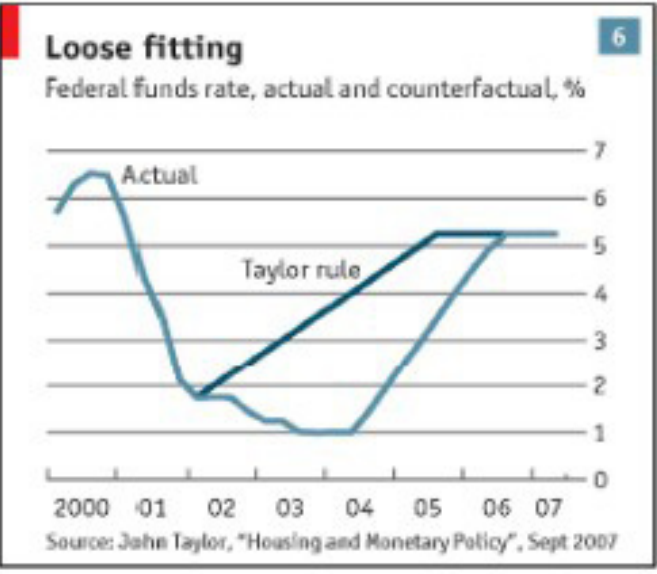

6. En relación a la transparencia y la publicidad obligatoria que requirió Basilea II, respecto a la información privilegiada (estructura de capital, metodología para las provisiones) que deben dar los bancos a sus interesados, tampoco se cumplió previamente a la gran crisis financiera norteamericana, ya que los bancos principales expusieron a grandes perdidas a sus confiados depositantes e inversores, ocultando información incluso a sus propios supervisores.

7. A partir de la llamada "consolidación de la banca” de 1998 en EE.UU, se deja expuesto a los intermediarios financieros que tenían una función delimitada en el ahorro y crédito comercial, a la tentación de incurrir por el desmesurado lucro personal (operadores con fuertes incentivos de cumplimiento) o lucro institucional, en el objetivo financiero de "maximizar el valor a los accionistas", ocasionando un riesgo descontrolado, involucrándose, como señalara el Premio Novel de Economía, Paul Krugman en entrevista al diario Gestión, en actividades de financiamiento de activos riesgosos de largo plazo y relativamente ilíquidos con deuda de muy corto plazo.

8. En este punto, relevándonos de mayor análisis, nos queda la convicción que adicional a la ineficiencia técnica de supervisión con que se venia manejando el sector bancario, existió grave infracción a la ética y otros valores, ya que adrede se expuso a grave riesgo el patrimonio personal de muchos clientes que les confiaron sus ahorros (llámesele depósitos, pequeñas inversiones etc.) y que en la debacle, no pudieron "salvar" nada. En esta tormenta financiera, como es conocida, también se afectaron muchos fondos de jubilación e instituciones benéficas.

\section{REFERENCIAS BIBLIOGRÁFICAS}

1. Banco Internacional de Pagos 2010 BIS: Basilea III: Marco regulador global para reforzar los bancos y sistemas bancarios. Bank for International Settlements Communications $\mathrm{CH}-4002$ Basilea, Suiza. ISBN version en línea: 92-9197-571-0

2 Heinrich Gregor (2005). Representante para las Américas del Banco de Pagos Internacionales (BIS). TÓPICO ESPECIAL 2: RETOS DE BASILEA II. ANALES ALIDE 33

3 Según John Taylor, ex-profesor de Economía de la Universidad de Columbia y alto funcionario de Bush Jr. en los primeros años de esta década, identifica la causa básica de la crisis sub-prime en la fijación de la tasa de interés de corto plazo. Note que entre 2002 y mediados de 2006, la tasa observada (actual) fue menor que la tasa sugerida por la regla de Taylor. O sea que en dicho período hubo una política de dinero fácil. 
2. -www.brettonwoodsproject.org.La arquitectura financiera internacional, 2006

3. Caputo Leiva, Orlando: La Economía Mundial a Inicios del Siglo XXI, 2007

4. Fernandez - Baca. Jorge. Dinero, Banca y mercados financieros. Centro de Investigación de la Universidad Pacífico, 2004.

5. Gómez Castañeda, O.R.:"Basilea I y II" en Observatorio de la Economía Latinoamericana No 56, febrero 2006. Texto completo en http://www.eumed.net/ cursecon/ecolat/ve/

6. Krugman, Paul: "Reforma financiera de Obama: saliendo de las sombras". Entrevista del diario Gestión del 24/04/2009.

7. Heinrich, Gregorio: Introducción a Basilea II: Fundamentos, áreas de discreción nacional y la ruta critica para su implementación / Congreso Bancario de Centroamérica, Panamá, República Dominicana y El Salvador, 13-15 de Abril del 2005.
8. Hennings Julio: Conferencia sobre la crisis financiera internacional / Colegio de Contadores Públicos de Arequipa, 2008.

9. Marthans Juan José, Examinando los riesgos macroeconómicos en Basilea II. Revista de temas financieros de la SBS, 2005.

10. Taylor John, Ensayo sobre la crisis. Revista Económica, Nov. 2008.

11. www.eleconomista.com.es/economía. Los bancos centrales del G-10 prevén que la economía mundial siga debilitándose, 2008

12. ws1. Grupo bbva. com sobre Basilea II, 2005.

13. Portal de la SBS: ¿Qué es Basilea III y por qué surgió?, 2011

14. Revista de la Superintendencia de Banca, Seguros y AFP del Perú: Preguntas sobre el Nuevo Acuerdo de capital, 2008. 